\title{
PD-L1 immunohistochemistry in patients with non-small cell lung cancer
}

\author{
Takanobu Jotatsu, Keishi Oda, Kazuhiro Yatera \\ Department of Respiratory Medicine, University of Occupational and Environmental Health, Kitakyushu, Fukuoka, Japan \\ Correspondence to: Keishi Oda, MD, PhD. Department of Respiratory Medicine, University of Occupational and Environmental Health, Japan, 1-1, \\ Iseigaoka, Yahatanishiku, Kitakyushu City, Fukuoka 807-8555, Japan. Email: oda-keishi@med.uoeh-u.ac.jp. \\ Provenance: This is an invited Editorial commissioned by the Section Editor Dr. Chunlin Ou (Cancer Research Institute of Central South University, \\ Changsha, China). \\ Comment on: Adam J, Le Stang N, Rouquette I, et al. Multicenter French harmonization study for PD-L1 IHC testing in non-small cell lung cancer. \\ Ann Oncol 2018;29:953-8.
}

Submitted May 09, 2018. Accepted for publication June 05, 2018.

doi: $10.21037 /$ jtd.2018.06.30

View this article at: http://dx.doi.org/10.21037/jtd.2018.06.30

Programmed death ligand 1 (PD-L1) is one of two ligands for programmed death 1 (PD-1) and can be expressed in lymphocytes ( $\mathrm{T}$ and $\mathrm{B}$ cells) and antigen-presenting cells, such as dendritic cells, macrophages, tumor cells and tumorinfiltrating immune cells. The PD-1/PD-L1 pathway plays an important role in the prevention of autoimmunity, and it has become clear that this pathway is one of the tumor immune escape mechanisms. By interrupting the interaction between PD-1 and PD-L1, the negative regulatory effects are reversed and the antitumor effect has been expected to be enhanced. Immune checkpoint inhibitors (ICIs) which are a type of immunotherapies have recently become a focus of attention in the field of oncology. Anti-PD-1 monoclonal antibodies and anti-PD-L1 monoclonal antibodies which are a kind of ICIs have improved the prognosis of patients with various types of cancer, and these drugs have already been applied in the clinical setting in the treatment of non-small cell lung cancer (NSCLC). In cases involving metastatic NSCLC and progression after platinum-based chemotherapy, pembrolizumab, which is one type of antiPD-1 monoclonal antibody was approved for the treatment of patients with NSCLC expressing PD-L1 in at least $1 \%$ of tumor cells based on data from the KEYNOTE-010 study (1). In patients with metastatic NSCLC and no prior systemic therapy, pembrolizumab was approved for the treatment of patients with NSCLC expressing PD-L1 in at least $50 \%$ of tumor cells on based on data from the KEYNOTE-024 study (2). In contrast, nivolumab which is another anti-PD-1 monoclonal antibodies was approved for all NSCLC, regardless of the PD-L1 expression, based on data from the CheckMate 057 (3) and CheckMate 017 (4) studies. Most recently, the anti-PD-L1 antibody atezolizumab was approved for the treatment of advanced NSCLC based on data from the OAK trial (5).

The PD-L1 expression of tumor cells is assessed by immunohistochemical staining of PD-L1. However, there is a major issue with regard to the assessment of the PD-L1 expression and the use of ICIs. To evaluate the PD-L1 expression, there are five different PD-L1 IHC assays. Each the ICIs has its own assay that was specifically developed and associated with a clinical trial. In the case of pembrolizumab, the PD-L1 IHC 22C3 pharmDx assay is performed on the Dako Autostainer Link 48 platform with an automated staining protocol using anti-PD-L1 antibody clone $22 \mathrm{C} 3$ to detect the expression of $\mathrm{PD}-\mathrm{L} 1$ protein, whereas the PD-L1 IHC 28-8 pharmDx assay is performed on the Dako Autostainer Link 48 platform using antiPD-L1 antibody clone 28-8 for nivolumab, the Ventana PD-L1 (SP263) assay is performed on the VENTANA Benchmark ULTRA staining platform with anti-PD-L1 antibody clone SP263 for durvalumab, and the Ventana PD-L1 (SP142) assay can only be performed using the VENTANA Benchmark ULTRA platform with anti-PD-L1 antibody clone SP142, an OptiView DAB IHC Detection Kit and an OptiView Amplification Kit for atezolizumab. The $22 \mathrm{C} 3$ assay is a companion diagnostic test, and the $28-8$ 
and SP142 assays are complementary diagnostic tests that are performed in the USA. In this way, it is recommended that each validated assay is performed on a particular staining platform. However, most diagnostic laboratories do not usually have two different automated staining platforms.

Recent report of Adam et al. (6) analyzed the concordance of staining results among five PD-L1 IHC antibodies (28-8, 22C3, E1L3N, SP142, and SP263) using the platforms that were available at different facilities. Previous studies raised concerns about the compatibility of the results from these IHC assays. Adam et al. showed the high concordance of the results of the assays using the 28-8, 22C3 and SP263 antibodies, consistent with the Blueprint Project Phase I results (7). Recent data presented by Fujimoto et al. (8) also showed that these assays have good concordance. The report from Adam et al. showed that an alternative assay performed with the antibody clone $22 \mathrm{C} 3$ on the Ventana staining platform had lower concordance than the Dako trial-validated assay (22C3 antibody clone on the Dako staining platform). A recent report by Neuman et al. (9) showed that two alternative assays with antibody clone 22C3 on the Ventana BenchMark XT platform demonstrated approximately $85 \%$ concordance with the results of the Dako trial-validated assay. With regard to the SP263 assay, it is known that there is a tendency for tumor cells and immune cells to be stained more strongly than is observed in the 28-8 and PD-L1 IHC 22C3 pharmDx assays (10). In the report by Adam et al. (6), Figure 2 showed that antibody clone 22C3 on the Ventana BenchMark UTLRA achieved a slightly lower positive rate in comparison to the study by Neuman et al. and that the SP263 antibody clone showed a high positive rate of 0.80 , which was higher than the rates observed with the other PD-L1 antibody clones among the seven centers. Recently, the SP263 assay has come into use as an alternative to both the $22 \mathrm{C} 3$ assay and the 28-8 assay for the evaluation of non-squamous cell NSCLC in Europe, based on the data from previous reports, such as the Blueprint Project Phase I (7), however, the decision with regard to non-squamous NSCLC patients was good because the Ventana platform is used by a greater number of laboratories than the Dako platform.

The SP142 assay was shown to stain fewer tumor cells in comparison to the 22C3, 28-8 and SP263 assays $(7,10)$. The data presented by Adam et al. (6) also showed that the positive rate in the SP142 assay tended to be lower than that in the SP263 assay. However, their SP142 assay data were compared to the SP263 assay on the Ventana platform; thus, we cannot judge their correctness. The poor staining of the tumor cells may be attributed to the protocols that were developed by the laboratory in which the amplification step is performed after antibody incubation and the use of a prediluted kit (approved by the Food and Drug Administration in the United States). A recent report in the European Society for Medical Oncology conference 2017 indicated that most tumor cells considered negative in PD-L1 IHC using antibody clone SP142 were also negative in IHC using the $22 \mathrm{C} 3$ antibody clone. Further data are required to judge whether the SP142 assay can be used as an alternative to the 22C3 PD-L1 IHC assay.

Antibody clone E1L3N is one of most popular antibodies in laboratory-developed tests (LDTs). A study compared three trial-validated assays (22C3, 28-8 and SP142) and an assay using antibody clone E1L3N as LDTs demonstrated that the 22C3 and 28-8 assays and the E1L3N LDT showed similar results with regard to staining tumor cells for PD-L1 (11). However, Adam et al. (6) reported that the E1L3N LDT displayed a relatively low positive rate for PD-L1 staining. They evaluated the concordance of SP142 and E1L3N assay compared with the SP263 assay. However, we considered that they should evaluate the concordance using various platforms with a PD-L1 IHC antibody.

Overall, the study by Adam et al. (6) provided valuable data on PD-L1 IHC testing, and the article was thoughtprovoking. Their conclusion was that $50 \%$ of the LDTs do not have sufficient concordance. The standardization of the staining process and platforms is important and urgent for obtaining consistent and reproducible results in the realworld clinical setting of lung cancer patients. In this article, the evaluation of PD-L1 staining was performed by one pathologist who had received comprehensive training in performing $28-8$ or $22 \mathrm{C} 3$ assays. The different PD-L1 IHC assays are not necessarily the same, and there is a possibility of different PD-L1 staining pattern. For example, the SP142 assay differs from other PD-L1 assays by its distinct staining pattern and stains both membrane and granular cytoplasm in tumor cells. Therefore, if a pathologist is only trained in who to perform $28-8$ or $22 \mathrm{C} 3$ assays, then he may not be trained to identify PD-L1 staining patterns using other PD-L1 IHC antibodies (SP142, SP263 and E1L3N). We conclude that PD-L1 staining should be evaluated by some pathologists.

Although these IHC assays identify the expression of PD-L1 protein, the antibody clones recognize different parts as the epitope of PD-L1 protein. In brief, the antibody clones 22C3 and 28-8 recognize the extracellular domain of the PD-L1 protein, and SP263, SP142 and E1L3N 
recognize the intracellular domain of PD-L1 protein. Furthermore, these antibody clones do not always display high affinity binding to these epitopes. The differences in these epitopes and in the binding affinity may lead to the different tumor cell staining results that are observed among the PD-L1 IHC antibody clones. It is also known that PD-L1 has two isoforms, and that the PD-L1 isoform expression might contribute to PD-L1 staining.

The importance of PD-L1 as a biomarker for anti-PD-1 antibodies or anti-PD-L1 antibodies cannot be ignored; however, there is an urgent need to search for another 'real' biomarkers that can predict the efficacy and safety of ICIs. ICIs will bring new hope to advanced NSCLC patients if a 'real' biomarker for ICIs can be discovered. Further efforts and studies for searching such biomarkers are also required.

\section{Acknowledgements}

None.

\section{Footnote}

Conflicts of Interest: The authors have no conflicts of interest to declare.

\section{References}

1. Herbst RS, Baas P, Kim DW, et al. Pembrolizumab versus docetaxel for previously treated, PD-L1-positive, advanced non-small-cell lung cancer (KEYNOTE-010): a randomised controlled trial. Lancet 2016;387:1540-50.

2. Reck M, Rodriguez-Abreu D, Robinson AG, et al. Pembrolizumab versus Chemotherapy for PD-L1Positive Non-Small-Cell Lung Cancer. N Engl J Med 2016;375:1823-33.

Cite this article as: Jotatsu T, Oda K, Yatera K. PD-L1 immunohistochemistry in patients with non-small cell lung cancer. J Thorac Dis 2018;10(Suppl 18):S2127-S2129. doi: 10.21037/jtd.2018.06.30
3. Borghaei H, Paz-Ares L, Horn L, et al. Nivolumab versus Docetaxel in Advanced Nonsquamous Non-Small-Cell Lung Cancer. N Engl J Med 2015;373:1627-39.

4. Brahmer J, Reckamp KL, Baas P, et al. Nivolumab versus Docetaxel in Advanced Squamous-Cell Non-Small-Cell Lung Cancer. N Engl J Med 2015;373:123-35.

5. Rittmeyer A, Barlesi F, Waterkamp D, et al. Atezolizumab versus docetaxel in patients with previously treated non-small-cell lung cancer (OAK): a phase 3, openlabel, multicentre randomised controlled trial. Lancet 2017;389:255-65.

6. Adam J, Le Stang N, Rouquette I, et al. Multicenter French harmonization study for PD-L1 IHC testing in non-small cell lung cancer. Ann Oncol 2018;29:953-8.

7. Hirsch FR, McElhinny A, Stanforth D, et al. PDL1 Immunohistochemistry Assays for Lung Cancer: Results from Phase 1 of the Blueprint PD-L1 IHC Assay Comparison Project. J Thorac Oncol 2017;12:208-22.

8. Fujimoto D, Sato Y, Uehara K, et al. Predictive Performance of Four Programmed Cell Death Ligand 1 Assay Systems on Nivolumab Response in Previously Treated Patients with Non-Small Cell Lung Cancer. J Thorac Oncol 2018;13:377-86.

9. Neuman T, London M, Kania-Almog J, et al. A Harmonization Study for the Use of 22C3 PD-L1 Immunohistochemical Staining on Ventana's Platform. J Thorac Oncol 2016;11:1863-8.

10. Scheel AH, Dietel M, Heukamp LC, et al. Harmonized PD-L1 immunohistochemistry for pulmonary squamouscell and adenocarcinomas. Mod Pathol 2016;29:1165-72.

11. Rimm DL, Han G, Taube JM, et al. A Prospective, Multi-institutional, Pathologist-Based Assessment of 4 Immunohistochemistry Assays for PD-L1 Expression in Non-Small Cell Lung Cancer. JAMA Oncol 2017;3:1051-8. 